\title{
Dissipation of jet bulk kinetic energy in powerful blazars
}

\author{
K. Katarzyński ${ }^{1,2}$ and G. Ghisellini ${ }^{1}$ \\ 1 Osservatorio Astronomico di Brera, via Bianchi 46, Merate and via Brera 28, Milano, Italy \\ e-mail: kat@astro.uni.torun.pl \\ 2 Toruń Centre for Astronomy, Nicolaus Copernicus University, ul. Gagarina 11, 87100 Toruń, Poland
}

Received 26 September 2006 / Accepted 20 October 2006

\section{ABSTRACT}

\begin{abstract}
Aims. We investigate the dissipation of the bulk kinetic energy of a relativistic jet at different distances from the central power-house and analyse in detail how the dissipated energy is radiated away.

Methods. We assume that the location of the dissipation region is a function of the bulk Lorentz factor $\Gamma$ of the jet, being closer to the centre for smaller $\Gamma$. This assumption is naturally fulfilled in the internal shock scenario. The dissipated energy is partially used to accelerate electrons and to amplify the magnetic field. This process creates a source inside the jet (blob). Such blobs may efficiently produce synchrotron and inverse Compton emission.

Results. We find that even if the blobs or shells responsible for the blazar activity carry the same energy (in bulk kinetic form), the fact that they move at different $\Gamma$ can produce dramatic variations in different bands, even if the bolometric luminosity is instead very similar. This is due to the relative importance of the synchrotron, self-Compton, and external Compton radiation processes, which greatly change by changing $\Gamma$ and the compactness of the source, even if the total radiated energy is constant. We then find that the jet can produce most of its radiative output at small distances from the putative black hole and its accretion disk, this implies a low level of emitted MeV-GeV flux. Our findings, which we apply for illustrative purposes to the blazar 3C 454.3, will be easily testable by the coming $\gamma$-ray satellite, such as AGILE and GLAST.
\end{abstract}

Key words. radiation mechanisms: non-thermal - galaxies: active - BL Lacertae objects: individual: 3C 454.3

\section{Introduction}

Our knowledge of the spectral energy distribution (SED) of blazars, at $\mathrm{MeV}-\mathrm{GeV}$ energies, has been built upon the results of the EGRET instrument onboard the Compton Gamma Ray Observatory, which shows that blazars as a class are powerful $\gamma$-ray emitters, and that the entire SED is characterised by two broad peaks, with the high energy one becoming more dominant for more powerful blazars (Fossati et al. 1998). This allows us to define a blazar sequence, whose main parameter is the bolometric observed luminosity, dictating the overall spectral appearance of the SED (Ghisellini et al. 1998): in more powerful objects, in which the radiative cooling is stronger, the emitting electrons have relatively small typical energies, explaining why in these sources the peak frequencies of the two peaks are smaller than in low power blazars. Since in these sources the stronger cooling is mainly due to the presence of external radiation (e.g., the broad emission lines), this scheme also explains why in powerful sources the high energy peak dominates. This blazar sequence has been obtained by averaging the data of a relatively large number of blazars (more than one hundred), and not all of them have been detected by EGRET. It then accounts for the fact that a sizeable fraction of blazars went undetected by EGRET. On the other hand, this average does not entirely account for the fact that one specific source can spend most of the time at $\mathrm{MeV}-\mathrm{GeV}$ fluxes that are below the detection threshold. Indeed, only a fraction (roughly $1 / 4$ ) of bright radio blazars were detected by EGRET. This can bias the representation of the average SED in the sense that the high energy flux might better represent the high state of the source, rather than its time-average SED. A related issue concerns the possibility of independent variability of the two peaks. Judging from the (few) examples we have, we know that variability at high energies (fluxes belonging to the high energy peak) is accompanied by flux variability at smaller (i.e., UV, optical, IR, but not radio) frequencies (with the rare but interesting exceptions of the socalled "orphan flares", i.e., TeV flux variations not accompanied by simultaneous X-ray variations; Krawczynski et al. 2004).

We then wonder if it is possible that a blazar flares in its synchrotron part of the spectrum, but not in the MeV-GeV band. As an illustrative example, consider the blazar 3C 454.3, detected by EGRET at the beginning of the nineties (Hartman et al. 1999), when the source was rather faint in the optical, and by BeppoSAX (in 2000, Tavecchio et al. 2002). The BeppoSAX spectrum, up to $\sim 100 \mathrm{keV}$, was relatively faint but very hard. Its extrapolation to the EGRET band was roughly consistent with the flux detected by EGRET in a different period of time. Recently, 3C 454.3 showed a huge flare in the optical (Fuhrmann et al. 2006; Pian et al. 2006) and in the X-ray bands, up to $100 \mathrm{keV}$, as detected by INTEGRAL and SWIFT (Pian et al. 2006; Giommi et al. 2006). However, we have no information about the emission level in the $\mathrm{MeV}-\mathrm{GeV}$ range during above mentioned activity. The SED of 3C 454.3 was interpreted by Ghisellini et al. (1998) and Tavecchio et al. (2002) as being due to a synchrotron and Inverse Compton model, produced by the one-zone region of the relativistic jet, in analogy with other powerful blazars. The high energy peak was the result of the inverse Compton scattering of relativistic electrons of seed photons coming from the broad line region (BLR), with the synchrotron self-Compton process playing a negligible role. If we apply the very same model to the optical/X-ray flaring state observed recently, then we are led to conclude that the $\mathrm{MeV}-\mathrm{GeV}$ 
emission should also show a flare, of similar amplitude to what seen in the optical ${ }^{1}$. We are then led to conclude that the jet has indeed changed its total power, by a large factor. Unfortunately, this scenario cannot be tested immediately due to lack of the $\mathrm{MeV}-\mathrm{GeV}$ observations. There are, however, more "economic" solutions, in which the total jet power changes much less, or is even constant. One solution was proposed in Pian et al. (2006): in this paper it was proposed that the emission site, responsible for the flare observed in 2005, was outside the BLR, where the number of external photons was negligible. In this case the external Compton component becomes negligible, and the power is mainly emitted by the synchrotron and self-Compton process. With roughly the same jet power, we can account both for the "high EGRET" state (with a large dominance of the high energy peak over the synchrotron one) and the "high synchrotron" state, where the power in the synchrotron and synchrotron-self Compton components are roughly equal, and equal to the previously observed "high EGRET" state.

In this paper we explore yet another possibility, in which the emission site is within the BLR, but the importance of the external Compton component is much reduced because of a reduced bulk Lorentz factor $\Gamma$. In fact, the importance of this component depends on the radiation energy density $U_{\text {ext }}^{\prime}$ of the seed external photons as seen in the comoving frame, and $U_{\text {ext }}^{\prime} \propto \Gamma^{2}$. It is then possible, in principle, to reduce the external Compton component and at the same time to enhance both the synchrotron and the self-Compton ones. To be more quantitative, we have to specify not only the $\Gamma$-factor, but also the size of the region, the magnetic field value, the density of the particles, and their energy spectrum. There is a specific scenario where all these quantities are all dependent in a specific way on the value of $\Gamma$, and this is the internal shock model. We will discuss it in detail in the next section, but the basic idea is that in this scenario the central engine is working intermittently, producing shells of matter moving at different speeds (and hence different Lorentz factors). At the start they are separated by same distance $\Delta R$, and there is always some probability that a later shell is faster then the previous one. The fastest shell will then catch up to the slower one, at a distance of the order of $R_{\text {coll }} \sim \Gamma^{2} \Delta R$. Therefore, if, on average, the two shells are produced with $\Gamma$ factors that are different, but relatively small, $R_{\text {coll }}$ will be correspondingly small and the emission region will be more compact, and the particle density and the magnetic field will be greater. If, in addition, we also assume that the kinetic and magnetic energy carried by the shells is always the same and that the dissipated energy (in the collision) is also the same, we determine that all quantities depend on $\Gamma$ only.

The internal shock scenario then offers a nice possibility to explore and quantify the consequences of the idea that a typical relativistic jet, even if it on average produces blobs of equal energies and therefore works, on average, at a constant power, it can still produce spectacular flares in different energy bands. However, we would like to stress that the main idea explored in this paper is not to test the internal shock scenario, but to investigate what happens if the jet, carrying on average the same amount of energy, dissipates it at different distances from the black hole, characterised by different $\Gamma$-factors (smaller $\Gamma$ at shorter distances). Our study also allows us to answer a more general question: Is it mandatory that the jet always produces most of the radiation we see at relatively large distances from the

${ }^{1}$ If the observed flux variation is due to an increase of the total number of electrons, then we expect, in this model, that variations of the synchrotron and of the external Compton components are linear. black hole? If the jet dissipation always results in the production of a dominant $\mathrm{MeV}-\mathrm{GeV}$ component the answer is yes. As discussed in Ghisellini \& Madau (1996), if high energy photons are produced too close to the accretion disk and its X-ray corona, they would be absorbed by photon-photon collisions, producing electron-positron pairs. These pairs would Compton scatter the UV-optical radiation from the disk, reprocessing the energy originally in the MeV-GeV band into the X-ray band. The observed "valley" between the two broad peaks of the blazar SED would then be filled by this reprocessed radiation. But what happens if the jet, close to the disk does not produce a too prominent $\mathrm{MeV}-\mathrm{GeV}$ component?

\section{The model}

In our model we assume a relativistic jet that contains electrons, protons, and a tangled magnetic field. Different sub-structures of this jet (hereafter shells) may travel with different velocities. Therefore, some of the shells may collide and generate relativistic shock waves. The shock, through the first order Fermi acceleration, may significantly increase the energy of the electrons. In other words, a fraction of the bulk kinetic energy of the colliding shells is used to accelerate electrons up to highly relativistic energies. The relativistic electrons escape from the shock front into the downstream region of the shock, where they lose their energy through the synchrotron and the inverse-Compton processes. These are the main assumptions of the so-called internal shock scenario, proposed for the first time by Rees (1978) and applied successfully for gamma-ray bursts (see, e.g., Meszaros 2006, for a recent review) and blazars (Sikora et al. 1994; Begelman et al. 1994; Ghisellini 1999; Spada et al. 2001; Guetta et al. 2004).

\subsection{Set up of the model}

In constructing our model we make two important assumptions. The first concerns the efficiency of the central engine that forms and accelerates the shells. We simply assume that the efficiency of the central engine is constant. This assumption is not a key ingredient of our model, but it allows us to see that even if the jet is working with the same efficiency, we can account for a large variation of the flux in different bands, as observed. According to this first assumption, the bulk Lorentz factor $\Gamma=1 / \sqrt{1-\beta^{2}}$ of the shell is inversely proportional to the total mass of the shell, so that the total energy given to the shell by the central engine is constant:

$M \Gamma=$ const.,

where $M \simeq N_{\mathrm{p}} m_{\mathrm{p}}\left(N_{\mathrm{p}}\right.$ is total number of protons of mass $\left.m_{\mathrm{p}}\right)$.

Consider now a collision of two shells ( $a$ and $b$ ) of bulk kinetic energy $\Gamma_{a} M_{a}$ and $\Gamma_{b} M_{b}$, respectively. The collision must satisfy energy

$\Gamma_{a} M_{a}+\Gamma_{b} M_{b}=\Gamma_{\mathrm{s}}\left(M_{a}+M_{b}+E^{\prime} / c^{2}\right)$

and momentum

$\Gamma_{a} \beta_{a} M_{a}+\Gamma_{b} \beta_{b} M_{b}=\Gamma_{\mathrm{s}} \beta_{\mathrm{s}}\left(M_{a}+M_{b}+E^{\prime} / c^{2}\right)$,

conservation, where $\Gamma_{\mathrm{s}}, \beta_{\mathrm{s}}$ describes the "merged" shell(s) created from the colliding shells and $E^{\prime}$ is the energy dissipated during the collision, measured in the comoving frame of the new shell. As mentioned above, this energy can be used to accelerate the electrons, and it can also be used for heating the protons or amplifying the magnetic field intensity. Since it is the new shell 
that contains the relativistic emitting particles, hereafter we will call it simply "source" or "blob". The efficiency describing how much of the kinetic energy of the two colliding shells is dissipated can be defined as

$\eta=\frac{E}{\Gamma_{a} M_{a}+\Gamma_{b} M_{b}}$,

where $E$ is the dissipated energy in the observer's frame. Using the conservation laws (Eqs. (2) and (3)) we can derive

$\eta=1-\frac{\Gamma_{\mathrm{s}}\left(1+\alpha_{M}\right)}{\Gamma_{a}\left(1+\alpha_{\Gamma} \alpha_{M}\right)}$,

where $\alpha_{M}=M_{b} / M_{a}$ and $\alpha_{\Gamma}=\Gamma_{b} / \Gamma_{a}$. Since our first assumption (shells of constant bulk kinetic energy) implies $\alpha_{\Gamma}=1 / \alpha_{M}$, the above formula simplifies into

$\eta \simeq 1-\frac{1+\alpha_{M}}{\sqrt{2+2 \alpha_{m}^{2}}}$,

(see, e.g., Lazzati et al. 1999). This simple relation shows that $\eta$ varies between 0 and $22 \%$ if the contrast between the Lorentz factors $\left(\alpha_{\Gamma}\right)$ changes from 1 to 10 .

Our second assumption concerns the amount of the energy transferred to the electrons during the collision. We simply assume that the electrons always receive the same amount of energy $\left(E_{\mathrm{e}}=\right.$ const.). Since our first assumption implies constant energy of the colliding shells, the present assumption requires only that the contrast between the Lorentz factors of the colliding shells is always the same ( $\alpha_{\Gamma}=$ const.). We are aware that this may be a great simplification of what we can expect in reality. However, we will see that this simple assumption is enough to provide a reasonable explanation for the some properties of blazar variability.

The total energy transferred to the electrons is given by

$E_{\mathrm{e}}=\Gamma E_{\mathrm{e}}^{\prime}=\Gamma N_{\mathrm{e}}\langle\gamma\rangle m_{\mathrm{e}} c^{2}=$ const.,

where $N_{\mathrm{e}}$ is the total number of electrons and

$\langle\gamma\rangle=\frac{\int_{\gamma_{\min }}^{\gamma_{\max }} \gamma Q(\gamma) \mathrm{d} \gamma}{\int_{\gamma_{\min }}^{\gamma_{\max }} Q(\gamma) \mathrm{d} \gamma}$

is the average electron energy, where $\gamma=1 / \sqrt{1-\beta_{\mathrm{e}}^{2}}$ is the electron random Lorentz factor and $Q(\gamma)$ describes the injection of the particles in the energy range constrained by $\gamma_{\min }$ and $\gamma_{\max }$. In the model we describe the particle acceleration as a continuous injection of relativistic electrons. This injection simulates the escape of the particles from the shock region into the downstream region of the shock that forms the source. First, we will discuss the very simple case of a monoenergetic injection, later we will discuss more complex, but more realistic, injections of power-law energy distributions. The duration of the injection is assumed to be equal to the source light crossing time $(R / c)$. During the process the electrons that are carrying the total energy described by Eq. (7) are injected into the source. The evolution of the particle energy distribution $N(\gamma, t)$ inside the source is calculated from the kinetic equation:

$$
\begin{aligned}
\frac{\partial N(\gamma, t)}{\partial t} & =\frac{\partial}{\partial \gamma}\left\{C \gamma^{2} N(\gamma, t)\right\}+Q(\gamma), \\
C & =\frac{4}{3} \frac{\sigma_{\mathrm{T}} c}{m_{\mathrm{e}} c^{2}}\left(U_{B}^{\prime}+U_{\mathrm{syn}}^{\prime}+U_{\mathrm{ext}}^{\prime}\right),
\end{aligned}
$$

where $U_{B}^{\prime}, U_{\text {syn }}^{\prime}, U_{\text {ext }}^{\prime}$ are the magnetic, synchrotron, and external radiation field energy densities, respectively. The solutions of this equation for the different types of injections that we use in our modelling were first derived by Kardashev (1962).

For the sake of simplicity, the source is approximated as a spherical homogeneous blob, of radius $R$ assumed to be proportional to the distance $D$ to the centre of the active nuclei ( $R=\psi D$, where $\psi$ is the jet aperture angle). We also assume that the $D$ depends on the bulk Lorentz factor $\Gamma$ as $D \propto \Gamma^{2}$. This assumption is supported by the internal shock model where two shells $\left(\Gamma_{b}>\Gamma_{a}\right)$ will collide when the second source $\left(\Gamma_{b}\right)$ will travel the distance

$D=2 \frac{\alpha_{\Gamma}^{2}}{\alpha_{\Gamma}^{2}-1} D_{0} \Gamma_{a}^{2}$,

where $D_{0}$ is the initial separation of the sources (see, e.g., Lazzati et al. 1999). In other words, relatively slow shells $(\Gamma \ll 10)$ may collide closer to the centre than faster shells $(\Gamma \gtrsim 10)$ that can collide only at larger distances. Note that this formula is valid only for constantly moving shells (no acceleration or deceleration). Moreover, this formula shows that our assumption $\left(D \propto \Gamma^{2}\right)$ becomes inaccurate for small $\Gamma$-contrasts $\left(\alpha_{\Gamma}<2\right)$. Finally, our assumption is supported by the fact that the central engine is probably not able to almost instantaneously accelerate the jet components up to very high values of $\Gamma$. If the acceleration process requires a time comparable to the travel time of the central engine (e.g., $0.1 \mathrm{pc}$ ) then we should expect fast shells farther from the centre than slow shells.

To complete the general description of our model we have to define the evolution of the magnetic field. By analogy to the definition of the energy transferred to the electrons (Eq. (7)), we assume that the total energy accumulated after the collision in the magnetic field, measured in the observer's frame, is constant

$E_{B}=\Gamma E_{B}^{\prime}=\Gamma U_{B}^{\prime} V=\Gamma B^{\prime 2} V^{\prime} /(8 \pi)=$ const.

where $B^{\prime}$ is the magnetic field intensity and $V^{\prime}$ is the source volume as measured in the comoving frame. In other words, the fraction of the total dissipated energy used for the amplification of the magnetic field is constant. Finally, some part of the dissipated energy can be transferred to the protons. However, we neglect this process in our computations since protons are not assumed to radiate and since their energy changes slightly for different Lorentz factors.

\subsection{Comparison of different sources}

Our main aim is to investigate how the energy transferred to the electrons (that is constrained to be exactly the same for every source) is radiated by different sources. By "different sources" we mean different states of the same jet. Therefore, we simply compare the emission of two sources with different Lorentz factors $\left(\Gamma_{2}<\Gamma_{1}\right)$. The difference in the Lorentz factors means that the sources were created by collisions of a pair of shells with different velocities (e.g., $\Gamma_{b}=20$ and $\Gamma_{a}=10 \rightarrow \Gamma_{1} \simeq 13.3$ and $\Gamma_{c}=8$ and $\Gamma_{d}=4 \rightarrow \Gamma_{2} \simeq 5.3$ ). Note that according to our assumption the contrast between the Lorentz factors of the colliding shells $\left(\alpha_{\Gamma}=\Gamma_{b} / \Gamma_{a}=\Gamma_{c} / \Gamma_{d}\right)$ is constant. Moreover, we assume that the shells before the collision contain only "cold" particles that cannot emit. Therefore, it is not mandatory to describe the physical parameters of both sources: it is sufficient to constrain the physical parameters of the first source using observations of the first state and derive the parameters of the second source (second state) choosing $\Gamma_{2}$ and using our assumptions

$R_{2}^{\prime}=R_{1}^{\prime}\left(\frac{\Gamma_{2}}{\Gamma_{1}}\right)^{2}, B_{2}^{\prime}=B_{1}^{\prime}\left(\frac{\Gamma_{2}}{\Gamma_{1}}\right)^{-3.5},\langle\gamma\rangle_{2}=\frac{N_{\mathrm{e}, 1}\langle\gamma\rangle_{1}}{N_{\mathrm{e}, 2}}$ 
Finally, to complete the description of the sources it is necessary to describe the injection function.

\subsection{Monoenergetic injection}

In the first approach we assume continuous monoenergetic injection of relativistic electrons

$Q(\gamma)=Q_{i} \operatorname{Dirac}\left(\gamma-\gamma_{i}\right)$,

where $Q_{i}$ is the number of injected particles per time unit and $\gamma_{i}$ describes the energy of the injected electrons. This approach helps to simplify the description of the model (e.g., $\left.\langle\gamma\rangle \equiv \gamma_{i}\right)$ and allows us to derive simple formulas that describe the evolution of the observed emission. According to the solution of the kinetic equation, the $N(\gamma)$ distribution is a power law

$N(\gamma)=\frac{Q_{i}}{C} \gamma^{-n}$ for $\gamma_{\min } \leq \gamma \leq \gamma_{i} ; \gamma_{\min }=\frac{\gamma_{i}}{1+C \gamma_{i} T}$,

where $n=2$ in this particular case, and $T^{\prime}=R / c$ is the duration time of the injection assumed to be equal to the source light crossing time. The total number of injected electrons is

$N_{\mathrm{e}}=V^{\prime} T^{\prime} \int Q(\gamma) \mathrm{d} \gamma$

that gives $N_{\mathrm{e}}=V^{\prime} T^{\prime} Q_{i}$ in the particular case of a monoenergetic injection. We assume a similar number of electrons and protons inside the shells before the collision (e.g., $N_{\mathrm{e}, \mathrm{a}} \simeq N_{p, a} \neq$ $\left.N_{\mathrm{e}, \mathrm{b}} \simeq N_{p, b}\right)$. However, only some fraction of the electrons may be accelerated. Moreover, we assume that the total number of accelerated electrons (total number of the injected electrons) is proportional to the total number of protons inside the source. According to our main assumptions $\left(\Gamma_{a} M_{a}=\Gamma_{b} M_{b}=\right.$ $\Gamma_{c} M_{c}=\Gamma_{d} M_{d}=$ const. and $\Gamma_{1} E_{1}^{\prime}=\Gamma_{2} E_{2}^{\prime}=$ const.) we obtain $\Gamma_{1}\left(M_{a}+M_{b}\right)=\Gamma_{2}\left(M_{c}+M_{d}\right)$. If we neglect the contribution of the electrons to the total mass of the shells then the total number of protons and therefore also the total number of electrons in different sources scales is

$N_{2}=N_{1} \frac{\Gamma_{1}}{\Gamma_{2}}$

This gives

$Q_{i, 2}=Q_{i, 1} \frac{V_{1}^{\prime} T_{1}^{\prime} \Gamma_{1}}{V_{2}^{\prime} T_{2}^{\prime} \Gamma_{2}}=Q_{i, 1}\left(\frac{\Gamma_{2}}{\Gamma_{1}}\right)^{-9}$,

where the injection time $\left(T^{\prime}\right)$ and the source volume $\left(V^{\prime}\right)$ scale is

$T_{2}^{\prime}=T_{1}^{\prime}\left(\frac{\Gamma_{2}}{\Gamma_{1}}\right)^{2}, \quad V_{2}^{\prime}=V_{1}^{\prime}\left(\frac{\Gamma_{2}}{\Gamma_{1}}\right)^{6}$.

Moreover, since $\gamma_{i} \equiv\langle\gamma\rangle$ the transformation of the total number of the particles gives $\gamma_{i, 2}=\gamma_{i, 1} \Gamma_{2} / \Gamma_{1}$. The electron density inside the source after the end of the monoenergetic injection is defined by

$K=\frac{Q_{i}}{C}=\frac{3 m_{\mathrm{e}} c^{2}}{4 \sigma_{T} c} \frac{Q_{i}}{U_{B}^{\prime}+U_{\mathrm{syn}}^{\prime}+U_{\mathrm{ext}}^{\prime}}$,

where the magnetic field energy density $\left(U_{B}^{\prime}\right)$ determines the synchrotron cooling during the injection, the synchrotron radiation field energy density $\left(U_{\text {syn }}^{\prime}\right)$ determines the cooling due to synchrotron self-Compton emission (this is correct only in the Thomson limit), and the radiation field energy density of the external medium, measured in the comoving frame of a source $\left(U_{\text {ext }}^{\prime}\right)$ determines the cooling due to inverse-Compton scattering of external photons. We can relatively easily describe the change of $U_{B}^{\prime}$ and $U_{\text {ext }}^{\prime}$ as a function of $\Gamma$. However, the precise description $U_{\text {syn }}^{\prime}$ is quite complex and requires a numerical approach. Therefore, for deriving some analytical formulas describing the emission processes, we will focus on two simple and opposite cases.

First, we assume the dominance of the EIC scattering $\left(U_{\text {ext }}^{\prime} \gg U_{B}^{\prime}\right.$ and $U_{\text {ext }}^{\prime} \gg U_{\text {syn }}^{\prime}$ ). Moreover, $U_{\text {ext }}^{\prime}$ is assumed to be constant in the observer's frame with a good approximation if this external radiation is produced by the Broad Line Region (BLR) (see e.g., Ghisellini \& Madau 1996). Therefore, the external radiation field density in the comoving frame for sources with different Lorentz factors scales is

$U_{\mathrm{ext}, 2}^{\prime}=U_{\mathrm{ext}, 1}^{\prime}\left(\frac{\Gamma_{2}}{\Gamma_{1}}\right)^{2}$.

This gives the following transformation of the particle density

$K_{2}=K_{1}\left(\frac{\Gamma_{2}}{\Gamma_{1}}\right)^{-11}$.

Knowing how all physical parameters of the source $\left(R^{\prime}, B^{\prime}, K\right.$, $\Gamma, U_{\text {ext }}^{\prime}$ ) scale with $\Gamma$, we can derive the approximated formulae describing the different types of emission, and how these depend on the bulk Lorentz factor. First, consider the synchrotron emission where the emissivity at a given frequency depends on the particle density and the magnetic field intensity as

$j_{\text {syn }}^{\prime}\left(v^{\prime}\right) \propto K B^{\prime 1+\alpha} v^{\prime-\alpha}$,

where $\alpha=(n-1) / 2$ is the spectral index. In the case of monoenergetic injection, $\alpha=0.5$. The observed thin synchrotron flux is given by

$F_{\text {syn }}(v) \propto \delta^{3+\alpha} R^{2} I_{\text {syn }}^{\prime}\left(v^{\prime}\right)$,

where $\delta=[\Gamma(1-\beta \cos \theta)]^{-1}$ is the source Doppler factor, $\theta$ is the viewing angle, and $I_{\text {syn }}^{\prime}\left(v^{\prime}\right) \propto R^{\prime} j_{\text {syn }}^{\prime}\left(v^{\prime}\right) \propto R^{\prime} K B^{\prime 1+\alpha} v^{\prime-\alpha}$ is the intensity of the thin synchrotron emission as measured in the comoving frame. For simplicity we neglect the self absorption regime (we will mainly investigate the synchrotron emission in the optical and X-ray energy bands where self-absorption is negligible). Moreover, to simplify the description even more we assume $\Gamma=\delta$. This is strictly true only if the viewing angle $\theta=1 / \Gamma[\mathrm{rad}]$. This assumption enables the possibility of describing the flux as a function of the Lorentz factor only. Strictly speaking this implies that when we compare the fluxes produced by sources with different Lorentz factors we compare these sources in different astrophysical objects with different viewing angles. However, this approximation does not qualitatively change our results in comparison to the case where we fix the viewing angle (this is mandatory when we study the same astrophysical object) and modify the value of the Doppler factor. Note that for $\theta=0$ the Doppler factor $\delta \sim 2 \Gamma$. We use this approach only for the tests presented in this subsection for illustrating the relations between different types of source emission in a simpler way. Using the above assumptions we can write $F_{\text {syn }}(v) \propto \Gamma^{3.5} R^{3} K B^{\prime 1.5} v^{-0.5}$ that gives the following scaling

$F_{\text {syn }, 2}(v)=F_{\text {syn }, 1}(v)\left(\frac{\Gamma_{2}}{\Gamma_{1}}\right)^{-6.75}$.

This shows that the synchrotron specific flux increases when $\Gamma$ decreases. This is because the increase of the magnetic field 
intensity and the particle density can easily compensate the decrease of source volume and the decreased Doppler boosting.

In a similar way we can describe the evolution of the selfCompton emission where the emissivity depends on the particle density and the intensity of the radiation field:

$j_{\text {SSC }}^{\prime}\left(v^{\prime}\right) \propto K I_{\text {syn }}^{\prime}\left(v^{\prime}\right) \propto R^{\prime} K^{2} B^{\prime 1.5} v^{\prime-0.5}$.

The observed flux is $F_{\mathrm{SSC}}(v) \propto \Gamma^{3.5} R^{4} K^{2} B^{\prime 1.5} v^{-0.5}$, which according to our previous assumptions scales is

$F_{\mathrm{SSC}, 2}(v)=F_{\mathrm{SSC}, 1}(v)\left(\frac{\Gamma_{2}}{\Gamma_{1}}\right)^{-15.75}$.

Note the stronger dependence on $\Gamma$ with respect to the synchrotron flux.

Finally, the external inverse-Compton (EIC) flux is given by $F_{\text {EIC }}(v)=F_{\text {EIC }, 0} v^{-0.5} \propto \Gamma^{3.5} R^{3} K I_{\text {ext }}^{\prime} v^{\prime-0.5}$. The (frequency integrated) intensity of the external radiation field in the comoving frame $\left(I_{\text {ext }}\right)$ is proportional to $\Gamma^{1.5}$. To understand this, assume a source with a constant size and particle density, surrounded by a constant external radiation field. Change only $\Gamma$. The total flux $\left(F_{\text {EIC }}^{\text {tot }}\right)$ produced by such a source is proportional to $\Gamma^{2} \delta^{4}$, and, according to our assumption $(\Gamma=\delta), F_{\text {EIC }}^{\text {tot }} \propto \Gamma^{6}$. Assuming a power-law spectrum of the emission we can write

$F_{\text {EIC }}^{\text {tot }}=\int_{v_{\min }}^{v_{\max }} F_{\text {EIC }, 0} v^{-0.5} \mathrm{~d} v \simeq F_{\text {EIC }, 0} v_{\max }^{0.5}$.

Since $v_{\max } \propto \delta \Gamma \sim \Gamma^{2}$, this relation gives $F_{\mathrm{EIC}, 0} \propto \Gamma^{5}$. Moreover, for the source where $R$ and $K$ do not depend on $\Gamma$ we have $F_{\text {EIC }}(v) \propto \Gamma^{3.5} I_{\text {ext }}^{\prime} v^{\prime-0.5} \propto \Gamma^{5}$ that gives $I_{\text {ext }}^{\prime}\left(v^{\prime}\right) \propto \Gamma^{1.5}$. Using this relation and the previous definitions we obtain

$F_{\mathrm{EIC}, 2}(v)=F_{\mathrm{EIC}, 1}(v)$,

as long as we compare the fluxes at a given frequency in the power law part of the spectrum. This means that our two sources with different Lorentz factors have the same specific flux if the total energy transferred to the particles is the same for each source and the physical parameters of the sources are scaled according to our prescriptions. This result is valid only for the sources where the emission is dominated by the external inverseCompton scattering.

Consider now the opposite case where the cooling is dominated by the synchrotron emission $\left(U_{B}^{\prime} \gg U_{\mathrm{ext}}^{\prime}\right.$ and $\left.U_{B}^{\prime} \gg U_{\mathrm{syn}}^{\prime}\right)$. The magnetic field energy density transforms as

$U_{B, 2}^{\prime}=U_{B, 1}^{\prime}\left(\frac{\Gamma_{2}}{\Gamma_{1}}\right)^{-7}$,

according to Eq. (20) gives

$K_{2}=K_{1}\left(\frac{\Gamma_{2}}{\Gamma_{1}}\right)^{-2}$.

This scaling differs significantly from the result obtained in the previous case (Eq. (22)), where $U_{\text {ext }}^{\prime}$ was dominant. Therefore, all formulae describing the different types of emission are now completely different. The synchrotron and SSC emission scale in the same way

$$
\begin{gathered}
F_{\mathrm{syn}, 2}(v)=F_{\mathrm{syn}, 1}(v)\left(\frac{\Gamma_{2}}{\Gamma_{1}}\right)^{2.25} \\
F_{\mathrm{SSC}, 2}(v)=F_{\mathrm{SSC}, 1}(v)\left(\frac{\Gamma_{2}}{\Gamma_{1}}\right)^{2.25} ;
\end{gathered}
$$

this implies a decrease of the flux when $\Gamma$ is decreasing. Also the EIC emission is decreasing with decreasing $\Gamma$

$F_{\mathrm{ext}, 2}(v)=F_{\mathrm{ext}, 1}(v)\left(\frac{\Gamma_{2}}{\Gamma_{1}}\right)^{9}$.

Then, when the synchrotron cooling is dominant, all three radiative processes produce an observed flux that decreases with decreasing $\Gamma$. Note that, in this context, a decrease of $\Gamma$ implies a comparison of two states of the jet, not a deceleration of a single source.

In Fig. 1 we compare the emission of different sources, located at different distances from black hole that are travelling with different velocities. In this test we assumed the following physical parameters for the fastest source: $\Gamma_{1}=20, R_{1}^{\prime}=2.4 \times$ $10^{16}[\mathrm{~cm}], B_{1}^{\prime}=7[\mathrm{G}], \gamma_{i, 1}=6 \times 10^{2}, Q_{i, 1}=8 \times 10^{-5}\left[\mathrm{~cm}^{-3} \mathrm{~s}^{-1}\right]$. We calculate the parameters for the other sources with $\Gamma<\Gamma_{1}$ according to our prescriptions. Moreover, we assume that the sources are travelling within a constant radiation field, produced by a spherical shell $\left(R_{\text {ext }}=3 \times 10^{17}[\mathrm{~cm}]\right)$. The emission of the shell is approximated as a black body with temperature $T_{\text {ext }}=2 \times 10^{4}[\mathrm{~K}]$ and peak luminosity $v_{\max } L_{\text {ext }}\left(v_{\max }\right)=$ $10^{45}\left[\operatorname{erg~s}^{-1}\right]$. We assumed a redshift $z=0.5$, and the Hubble constant $H_{0}=72\left[\mathrm{~km} \mathrm{~s}^{-1} \mathrm{Mpc}^{-1}\right]$. Since we will later apply our model to low energy peaked blazars the chosen parameters are somehow representative of these clases of objects.

The reason why we parametrise the fastest source and not the slowest one is purely "technical". To calculate the particle distribution after the end of the injection we have to know the values of $U_{B}^{\prime}, U_{\text {syn }}^{\prime}$, and $U_{\text {ext }}^{\prime}$. The values of $U_{B}^{\prime}$ and $U_{\text {ext }}^{\prime}$ can be calculated straightforwardly from our parametrization, but the estimate of $U_{\text {syn }}^{\prime}$ is more complex, since it depends on $N(\gamma)$, which in turn depends on $U_{\text {syn }}^{\prime}$. We then proceed by calculating the spectra for sources with different Lorentz factors in a sequence, decreasing the value of $\Gamma$ by a very small factor. This allows us to use the value of $U_{\text {syn }}^{\prime}$ from the previous step in the current computations if the change of $U_{\text {syn }}^{\prime}$ from one step to another is small. This method requires that in the first step the synchrotron radiation field energy density is negligible with respect to the other energy densities. Since (for our set of parameters) the fastest source cools mainly by the external radiation field, we always start the computations from the largest value of $\Gamma$.

Our set of input parameters implies $U_{\text {ext }}^{\prime} \gg U_{B}^{\prime} \gg U_{\text {syn }}^{\prime}$ for the fastest source (Fig. 1d). Therefore, in agreement with our analytical predictions, initially $F_{\text {EIC }}(v)$ remains constant, whereas $F_{\text {syn }}(v)$ and $F_{\text {SSC }}(v)$ are increasing fast for decreasing $\Gamma$ (Fig. 1c). However, the decrease of the Lorentz factor quickly reduces the importance of the external radiation field in the comoving frame. Thus, the synchrotron emission becomes the dominant cooling process (Fig. 1d). This leads to a fast decrease of $F_{\text {EIC }}(v)$ and also causes the decrease of $F_{\text {syn }}(v)$ and $F_{\text {SSC }}(v)$, as predicted (Fig. 1c). Note that the parameters chosen for this particular simulation gives a very weak contribution of the SSC process to the total emission that appears visible only in the sources with very small $\Gamma$ values (Fig. $1 b$ ).

In the second test (Fig. 2) we modified some of the physical parameters $\left(R_{1}^{\prime}=2.4 \times 10^{16} \rightarrow 7.5 \times 10^{15} \mathrm{~cm}, B^{\prime}=7 \rightarrow 5 \mathrm{G}\right.$, $Q_{i, 1}=8 \times 10^{-5} \rightarrow 1.5 \times 10^{-2} \mathrm{~cm}^{-3} \mathrm{~s}^{-1}$ ) to amplify the SSC emission. However, even for this new set of the parameters the SSC and synchrotron cooling are negligible in the fastest source $\left(\Gamma_{1}=20\right)$ where the EIC emission dominates as in the previous test (Fig. 2a). As we already mentioned, the EIC cooling decreases quickly with the decrease of $\Gamma$ at the advantage of other cooling processes. In this particular case, when $\Gamma \sim 10$, the 

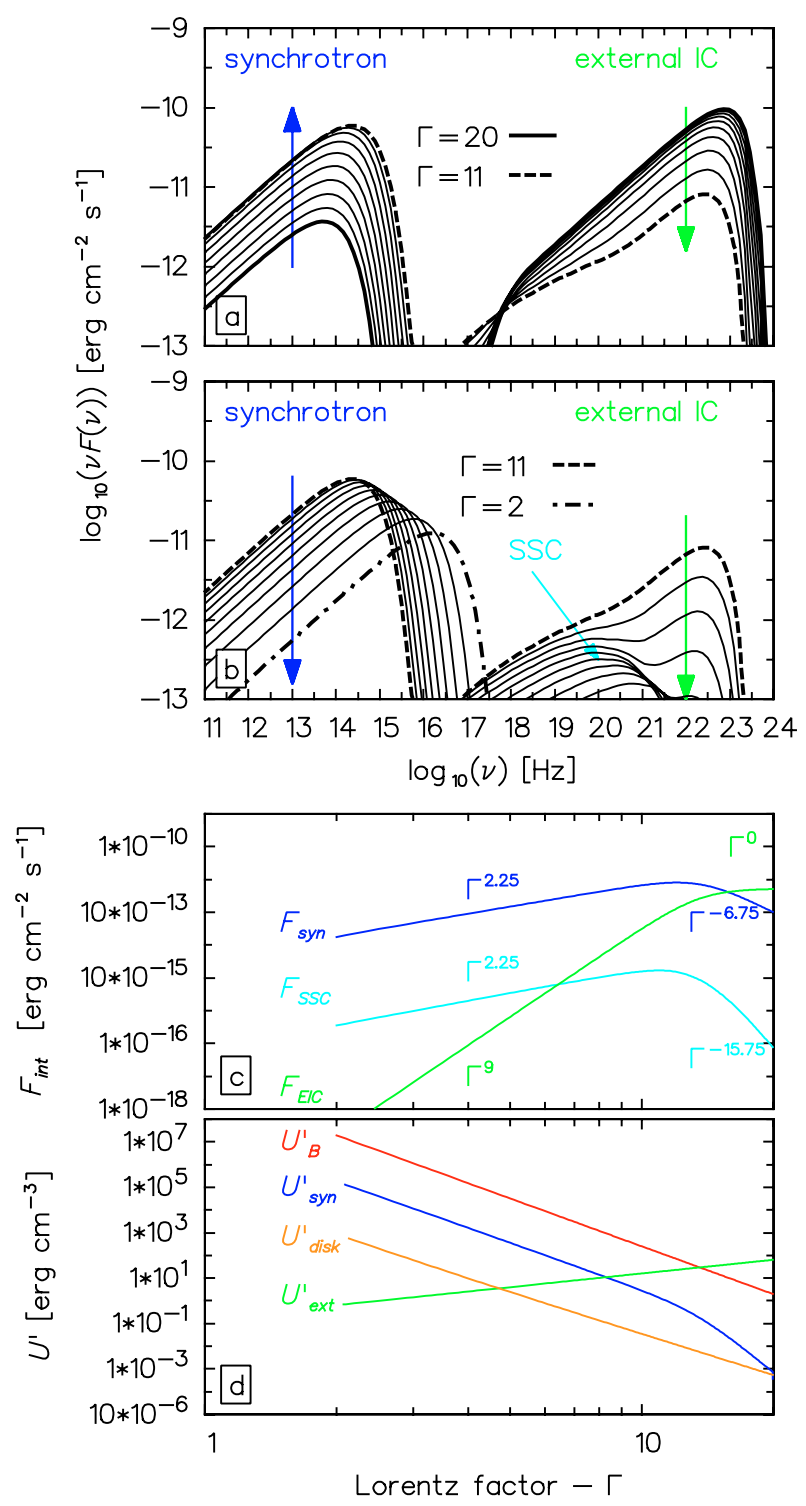

Fig. 1. In the upper panels we compare total emission of sources located at different distances from the centre and travelling with different bulk Lorentz factors $(\Gamma=20 \rightarrow 11$ panel a), and $\Gamma=11 \rightarrow 2$ panel b)). The total energy transferred to the electrons is exactly the same for each source. In this particular case the electrons are injected with a monoenergetic distribution. Panel c) shows, separately, the fluxes of the different emission mechanisms, integrated over narrow frequency ranges: synchrotron $\left(10^{11}-10^{12} \mathrm{~Hz}\right), \operatorname{SSC}\left(10^{14}-10^{15} \mathrm{~Hz}\right)$ and the external inverse-Compton emission $\left(10^{19}-10^{20} \mathrm{~Hz}\right)$. In the lower panel d) we compare the different types of the energy densities that control the particle cooling. This shows that, in this particular simulation, the external inverse-Compton scattering dominates the cooling process for large values of $\Gamma$ whereas the synchrotron cooling is dominating for $\Gamma<10$.

synchrotron and SSC cooling processes become dominant and almost equally important $\left(U_{B}^{\prime} \sim U_{\text {syn }}^{\prime} \gg U_{\text {ext }}^{\prime}\right.$, Fig. 2d). Moreover, the second order SSC emission also appears significant in this particular test. However, the energy density of the SSC emission $\left(U_{\text {SSC }}^{\prime}\right)$ that describes the particle cooling due to 2 nd order SSC is for all the sources a few times smaller than $U_{B}^{\prime}$ (Fig. 2c). Note that if the SSC emission were stronger than the synchrotron radiation $\left(U_{\text {syn }}^{\prime}>U_{B}^{\prime}\right)$, the 2nd order SSC scattering would occur in the Klein-Nishina regime. This would require a special treatment of the cooling process and a numerical solution of the kinetic equation. Our parameter choice is
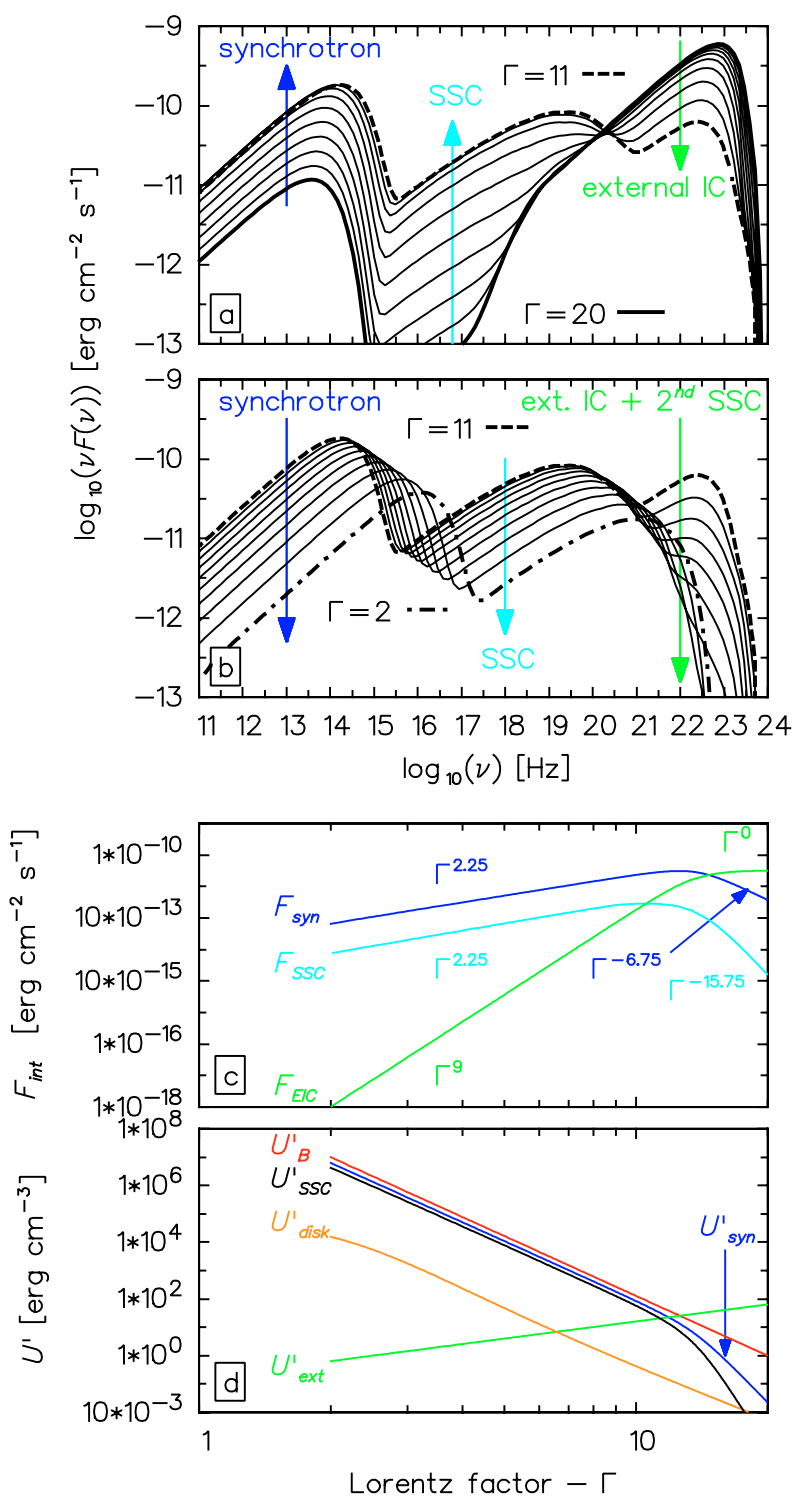

Fig. 2. Top panels: comparison between spectra produced by different sources in the case of monoenergetic injection. In this particular test the SSC emission appears almost as important as the synchrotron radiation for sources with $\Gamma \sim 10$, for which the efficiency of the EIC process is significantly reduced. Note that the second order SSC process also appears significant in this particular case. The spectra of the different emission processes are presented in Fig. 3 for three representative sources. Bottom panels: as in Fig. 1, it is shown how the fluxes of the different emission processes, and the corresponding energy densities, scale as a function of $\Gamma$.

such that $U_{\text {syn }}^{\prime}<U_{B}^{\prime}$ in all cases. As a consequence, the numerical calculations are always in perfect agreement with the analytic predictions. In Fig. 3 we show, separately, the spectra produced by the different emission mechanisms, and how their importance changes varying $\Gamma$. The fastest source $(\Gamma=20)$ is emitting the dominant part of the available energy through the EIC scattering. The moderately fast source $(\Gamma=11)$ uses the synchrotron and the SSC processes to radiate the particle energy. The EIC in this source is significantly less important, producing a flux of the same order as the second order SSC emission. Finally, the slowest source $(\Gamma=2)$ is producing mostly synchrotron and SSC radiation. The parameters of the sources span the ranges: $R^{\prime}=8 \times 10^{15} \rightarrow 8 \times 10^{13} \mathrm{~cm}$, 


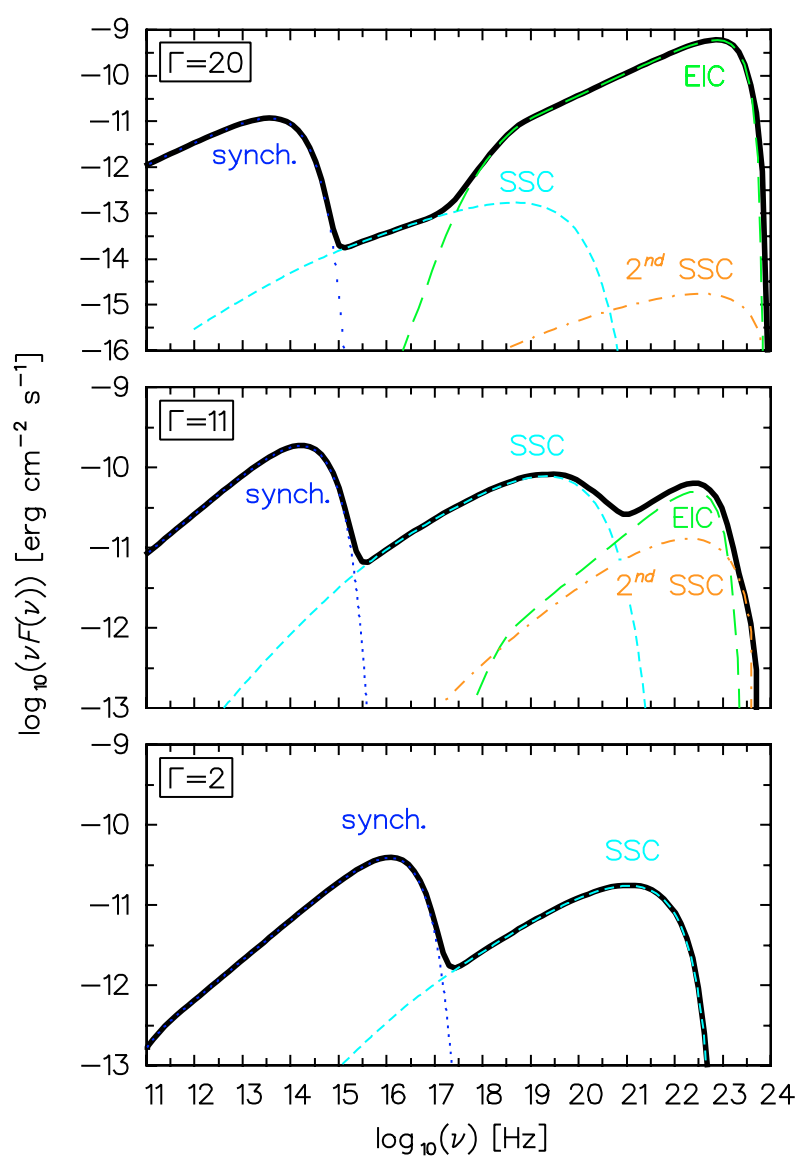

Fig. 3. The components in the total spectrum produced by three different sources characterised by different values of $\Gamma$. The spectra are chosen from the sequence presented in Fig. 2. In the fastest source $(\Gamma=20)$ the external inverse-Compton emission (EIC, long dashed line) appears dominant. In the moderately fast source $(\Gamma=11)$ the synchrotron (dotted line), SSC (short-dashed line), and the EIC processes are producing similar amounts of radiation. Moreover, the second order SSC process (dash-dot line) is also not negligible for this case. A relatively slow source $(\Gamma=2)$ is producing mostly synchrotron and SSC emission.

$B^{\prime}=5 \rightarrow 1.6 \times 10^{4} \mathrm{G}$, and $K=7.1 \times 10^{3} \rightarrow 2.3 \times 10^{7} \mathrm{~cm}$. In all cases we calculate the optical depth for the pair production process inside the source due to the interaction between $\gamma$-rays and lower energy photons. This process appears negligible for all sources.

We also estimated the energy density of the radiation produced by the accretion disk ( $U_{\text {disk }}^{\prime}$ ) in the comoving frame according to the prescription of Ghisellini \& Madau (1996). For this estimate, we assume that the total luminosity of the disk is ten times greater than the luminosity of the external radiation field, which is a good approximation if we assume that the external radiation field is produced by the broad line region. The distance between the putative black hole and the source $(D)$ is calculated assuming that the jet is conical, with an aperture angle of $0.1 \mathrm{rad}(\sim 5$ degrees $)$, giving $D=R / 0.1$. The disk energy density starts to dominate over $U_{\text {ext }}^{\prime}$ at relatively small distances from the centre, corresponding to small $\Gamma$-values $(\Gamma \lesssim 5)$. Nevertheless, $U_{\text {disk }}^{\prime}$ always remains at least two orders of magnitude weaker than the energy density of the magnetic field and the internal radiation fields $\left(U_{\mathrm{syn}}^{\prime}\right.$ and $\left.U_{\mathrm{SSC}}^{\prime}\right)$. Therefore, the disk radiation field is negligible as a cooling agent of the particles inside the source.
The above tests show that according to our prescription the fast moving sources radiate away the dissipated energy through the inverse-Compton scattering of the external photons, whereas the slow moving sources may efficiently produce synchrotron and also SSC emission. The relative level between the SSC and the synchrotron emission depends on the physical parameters of the source. What is important is that the same amount of the dissipated energy is radiated away, but with different emission processes. We can then observe completely different spectra originating in the same jet, according to whether the dissipation takes place in its inner (small $\Gamma$-values) or outer (large $\Gamma$ ) part. For instance, the same cosmic source can display a state with a moderate optical synchrotron emission and a strong $\gamma$-ray EIC radiation (at $\mathrm{MeV}-\mathrm{GeV}$ energies), or a strong optical emission and a strong SSC radiation (at $\mathrm{keV}$ energies) without a corresponding higher level of the MeV-GeV flux. Dramatic variability of a particular object would not correspond to dramatic variations on the jet efficiency (we have assumed that the blobs always carry the same energy), but would rather reflect the place, along the jet, where dissipation is taking place (if this location is in turn linked with the bulk Lorentz factor).

Our model is rather "economic" for the jet, in the sense that the total energy radiated during different flares is approximately the same, even if the variations in specific bands can be dramatic. The fact that a synchrotron (in the IR-optical band) and an X-ray flare may not be accompanied by a simultaneous flare in the $\mathrm{MeV}-\mathrm{GeV}$ band is the main prediction of our model, which can be easily verified by the new $\gamma$-ray observatories (GLAST, AGILE). These kinds of observations may prove if the jet is varying its (likely kinetic, but possibly also magnetic) power or if it is instead working at a constant average efficiency.

The monoenergetic injection used in our test provides a very simple description of the model and allows us to describe the scaling of different radiative processes in an analytic way. However, such a simple injection is corresponding to a particle distribution, which is a power law, of slope $n=2$, which cannot accurately explain most of the observations. Therefore, in the next step we discuss the injection of a power-law particle distribution.

\subsection{Injection of a power-law electron distribution}

In a homogeneous source, the spectrum of the observed emission depends directly on the shape of the particle distribution. Therefore, to precisely reproduce the observed spectra of blazars, we need a particle energy spectrum that is more complex than a single power law provided by the monoenergetic injection. Quite a large flexibility in spectral fitting is obtained if we assume a power-law energy distribution of the injected particles

$Q(\gamma)=Q_{\mathrm{i}} \gamma^{-n}$, for $\gamma_{\mathrm{i}} \leq \gamma \leq \gamma_{\max }$

For $\gamma_{\mathrm{i}}>1$ and $n>2$ such injection with the simultaneous radiative cooling provides a particle distribution that can be approximated by two power-law functions

$N(\gamma)=\left\{\begin{array}{ll}K \gamma^{-2} & \text { for } \gamma_{\min } \leq \gamma \leq \gamma_{\mathrm{i}} \\ K_{2} \gamma^{-(n+1)} & \text { for } \gamma_{\mathrm{i}}<\gamma \leq \gamma_{\max }\end{array}\right.$,

where $K_{2}=K \gamma_{i}^{n-1}$ and the particle density $(K)$ is directly related to the injection rate $\left(Q_{\mathrm{i}}\right)$ and the cooling $(C)$ efficiency

$K=\frac{Q_{\mathrm{i}}}{C(n-1) \gamma_{\mathrm{i}}^{n-1}}$ 

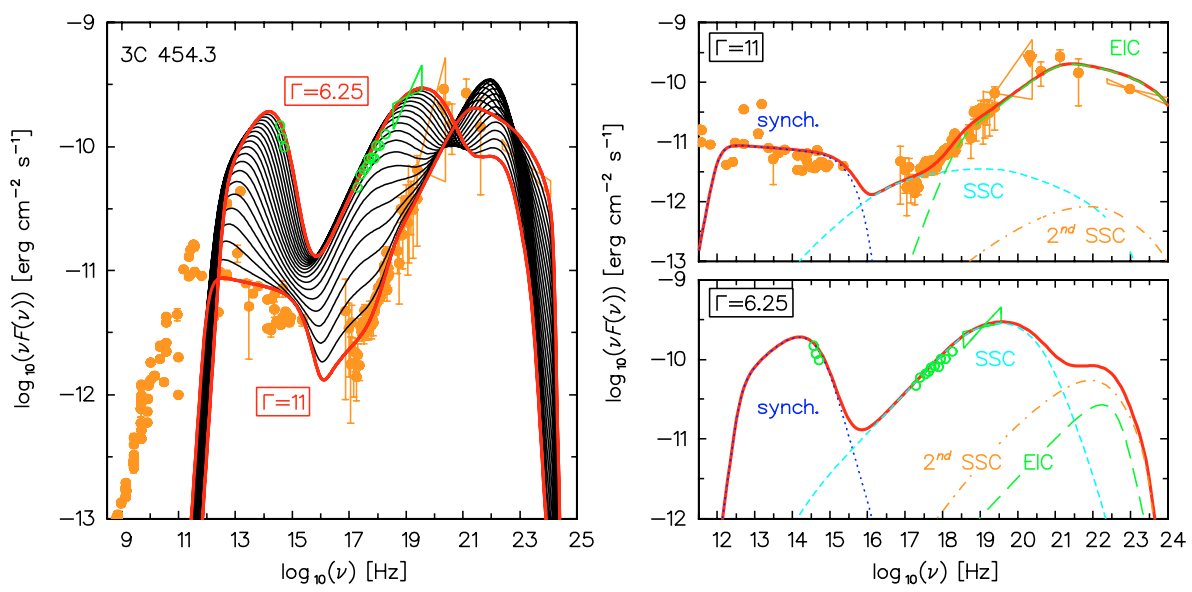

Fig. 4. The multifrequency observations of 3C 454.3 for two different emission states together with the results of our modelling. The low level of the optical and X-ray emission (state 1 ) is represented by data (filled circles) obtained at different epochs (references to the data points in the text) whereas the high synchrotron and X-ray state (state 2) is represented by the observations made by the Swift satellite and the REM telescope (Giommi et al. 2006). The left panel shows a sequence of spectra with different Lorentz factors: the model with the largest $\Gamma$ reproduces state 1 and the model with the smallest $\Gamma$ can explain state 2 . In the right panels we show how the different emission processes contribute to the total spectrum.
The minimum energy $\gamma_{\min }$ is calculated in the same way as for the monoenergetic injection (Eq. (15)), whereas $\gamma_{\max }$ is a free parameter, whose value is not important if $n>2$. The injection rate can be calculated from the simple relation

$Q_{\mathrm{i}}=\frac{N_{\mathrm{e}}}{T^{\prime} V^{\prime}\left(\gamma_{\max }^{1-n}-\gamma_{\mathrm{i}}^{1-n}\right) /(1-n)}$,

where $N_{\mathrm{e}}$ is the total number of injected particles.

We will assume that sources with different $\Gamma$-values may have different slopes and different energy ranges of the injection, but we still maintain our main assumption on the total energy of the injected electrons, which must be the same for all sources (Eq. (7)). This gives the following relation between the total number of particles, average energy, and $\Gamma$ in two different sources

$N_{\mathrm{e}, 2}=N_{\mathrm{e}, 1} \frac{\langle\gamma\rangle_{1} \Gamma_{1}}{\langle\gamma\rangle_{2} \Gamma_{2}}$

Using the above description we can calculate the emission from different sources assuming different injection profiles but using the same amount of the injected energy. Even if the power-law injection is more realistic than the monoenergetic injection, the results obtained are qualitatively the same for both cases. We will directly apply our simulations, done for a power-law injection case, to the recent observations of the blazar 3C 454.3, which we will use as an illustrative example.

\section{Application to $3 \mathrm{C} \mathbf{4 5 4 . 3}$}

Recent observations of the blazar 3C 454.3 showed a huge flare in the optical and X-ray bands (Fuhrmann et al. 2006; Pian et al. 2006; Villata et al. 2006; Giommi et al. 2006). We will investigate if such activity is in agreement with the prediction of our model, in which sources with relatively small velocities $(\Gamma \lesssim 10)$ can produce mostly synchrotron (IR $\rightarrow$ UV) and SSC (UV $\rightarrow$ hard X-ray) emission. Therefore in the next step we will try to reproduce this unusual activity.

In Fig. 4 we collected the multifrequency observations of 3C 454.3. Most of the data are historical observations made at different epochs, illustrating the low level of the optical and $\mathrm{X}$-ray emission. The historical data from the radio frequencies up to the optical range are mostly taken from the NED database. However, in this range we are also plotting a few points from other sources (see, Pian et al. 2006, and references therein). In the X-ray range we show the low/hard emission level observed by BeppoSAX in 2000 (Tavecchio et al. 2002). Finally, in hard
X-rays and $\gamma$-rays we show the COMPTEL and EGRET measurements made in the years 1991-1994 (Zhang et al. 2005; Hartman et al. 1999). The high level of the optical and X-ray emission is shown by the data taken simultaneously by three experiments (UVOT, XRT, BAT) onboard the Swift satellite and by the quasi-simultaneous ground-based data taken by the REM telescope (Giommi et al. 2006). We will focus onto two states of the source: state 1 corresponds to the high level of $\gamma$-ray emission, low/hard X-ray flux, and low IR-optical flux, while state 2 corresponds to the recent optical and X-ray flare. Note that position of the synchrotron and the IC peak are not well determined for the second state.

We first try to reproduce state 1 . Assuming that the $\gamma$-rays are produced by the EIC scattering inside a relatively fast moving source, we obtained the best fit using the following parameters: $\Gamma_{1}=\delta_{1}=11, R_{1}^{\prime}=1.1 \times 10^{16} \mathrm{~cm}, B_{1}^{\prime}=4.8 \mathrm{G}, \gamma_{\mathrm{i}, 1}=90, \gamma_{\max }=$ $5 \times 10^{3}, Q_{\mathrm{i}, 1}=25.4 \mathrm{~cm}^{-3} \mathrm{~s}^{-1}, n_{1}=2.1$. For the external radiation field we used $v_{\max } L_{\text {ext }}\left(v_{\max }\right)=10^{45} \mathrm{erg} \mathrm{s}^{-1}, T_{\text {ext }}=2 \times 10^{4} \mathrm{~K}$, $R_{\text {ext }}=2.8 \times 10^{17} \mathrm{~cm}$. The spectral index of the injected particles is relatively $\operatorname{soft}\left(n_{1}=2.1\right)$, to explain the soft spectrum observed in the IR-UV range and also the relatively soft spectrum in the $\mathrm{MeV}-\mathrm{GeV}$ range observed by EGRET.

To reproduce state 2 we calculate a sequence of spectra decreasing the value of $\Gamma(\Gamma=11 \rightarrow \Gamma=6.25)$ by steps of $\Delta \Gamma=0.25$. Moreover, in each step we increase the spectral index of the injected particle distribution by a factor $\Delta n=1 / 3$. Also the minimum energy of the injected particles $\gamma_{i}$ increases by a factor $\Delta \gamma_{i}=0.6 \gamma_{i, 1}$ whereas $\gamma_{\max }$ is assumed to be constant. According to our main assumption the changes of the injection spectrum are compensated by the change in the normalisation (Eq. (37)) to provide the same amount of the injected energy for all states. Note that in this case we maintain the viewing angle fixed $\left(\theta=5.2^{\circ}\right)$ and therefore, in general, $\delta \neq \Gamma$. The best fit for state 2 is reached for $\Gamma_{2}=6.25, \delta_{2}=9.42, R_{2}^{\prime}=3.7 \times 10^{15} \mathrm{~cm}$, $B_{2}^{\prime}=35.7 \mathrm{G}, \gamma_{\mathrm{i}, 2}=6 \times 10^{2}, Q_{\mathrm{i}, 2}=3.4 \times 10^{13} \mathrm{~cm}^{-3} \mathrm{~s}^{-1}, n_{2}=5.26$. Note that while the set of parameters used for state 1 was chosen to best represent the data, the parameters for state 2 are given by our assumed parametrization, and are not free. Note also that here we appropriately calculate the synchrotron self-absorbed spectra that we previously neglected.

Self-absorption truncates the synchrotron spectrum below the IR range. This effect appears frequently in the modelling of the high energy emission of blazars and is related to the size of the source that must be relatively small $\left(10^{15 \rightarrow 16}[\mathrm{~cm}]\right)$ to explain the observed variability time scales. Such a small source must be also relatively dense (both in particles and magnetic field) to explain the observed emission level and therefore it is 
optically thick at radio frequencies. The radio emission should be produced by more extended radio structures, well visible in the VLBI maps. Moreover, this particular blazar does not show variability of the radio flux clearly correlated with the optical, $\mathrm{X}$-ray, and $\gamma$-ray activity (e.g., Villata et al. 2006). This suggests that the quiescent radio and the variable optical-to- $\gamma$-ray emission are produced in different regions of the source. At any rate, the analysis of the radio emission probably produced by an inhomogeneous jet is out of the scope of this work.

Note that our model reproducing state 2 is very similar to the spectrum resulting from the modelling (of the same state) made by Pian et al. (2006). In that paper, the emitting region was assumed to be outside the broad line region, to neglect the external radiation and the corresponding external Compton emission. To compensate, the relatively low values of the magnetic field and the particle density, appropriate at such large distances from the centre, it was necessary to adopt a large $\Gamma$, giving a Doppler factor $\delta=15$. This difference in the $\Gamma$-factors gives a potential tool to discriminate between the model presented here (which has $\delta=9.4$ ) and the model discussed in Pian et al. (2006). In fact, in our model, the apparent velocities of VLBI knots (after an optical and X-ray flare) should be smaller than in the Pian et al. 2006 model. Unfortunately, the predicted differences are not large, and probably not measurable with enough accuracy for this particular blazar.

The presented simulation shows that our approach can well explain two different levels of the emission observed in $3 \mathrm{C}$ 454.3. We stress that what we proposed is the most "economic" way to reproduce dramatically different levels of emission, even if the jet is using always the same amount of energy. The strong prediction of this approach is that the level of the $\gamma$-ray MeV-GeV emission should be lower (or not particularly bright) when the level of the IR-to-X-ray emission is high. For the moment there is no evidence of such behaviour. We must wait for simultaneous optical, X-ray, and especially $\gamma$-ray observations of blazars to verify if our idea is correct.

\section{Summary and conclusions}

We have proposed a model to explain different levels of the emission observed from the IR range up to the $\gamma$-ray band in a powerful blazars. The main assumptions of our model are that the emission zone of the jet is always dissipating the same amount of energy, but that the emission zone can be located in different parts of the jet, farther from the central engine if the bulk Lorentz factor is larger. We have discussed a particular scenario where this naturally occurs, namely the internal shock scenario. While this scenario is not mandatory to develop our main idea, it is the only existing scenario where we can work out how all the relevant quantities (emission volume, magnetic field, particle density, and so on) scale with the bulk Lorentz factor $\Gamma$. The obtained results are certainly promising, since even with our simplifying assumption (the emission sites always dissipate the same amount of total energy) we were able to explain dramatically different states observed at different frequency bands. In particular, we were able to explain two very different states of the blazar 3C 454.3. This is achieved by noting that when the bulk Lorentz factor $\Gamma$ is small, the shell-shell collision in the internal shock scenario occurs closer to the apex of the jet, therefore in a more compact region, with a larger magnetic field. Since the external Compton radiation is dimmed in this case (being less boosted in the comoving frame), the electron energy is radiated more through the synchrotron and the self-Compton processes, therefore not at $\mathrm{MeV}-\mathrm{GeV}$ energies, but in the IR-optical (through synchrotron) and X-rays (through self-Compton). Lowering the $\Gamma$-factor there is therefore a "transfer of power" from higher to lower frequencies. If this idea is correct, there should exist sources flaring in the optical and X-ray bands, but not in $\gamma$-rays, where the flux can even decrease. This prediction can be easily tested by the forthcoming $\gamma$-ray satellites, AGILE and GLAST, together with simultaneous optical and X-ray observations: had $\mathrm{MeV}-\mathrm{GeV}$ observations been available during the 2005 flare of 3C 454.3, such a test could have already been done.

The possibility that the jet can dissipate part of its bulk kinetic energy at small distances from its apex, and at small distances from the accretion disk, may at first sight contradict what was claimed in Ghisellini \& Madau (1996), where strong dissipation close to disk was excluded. But in that case "dissipation" meant strong production of $\gamma$-rays, dominating the radiative output. Here, instead, when the dissipation occurs "early" (i.e., close to the disk) the external Compton flux is unimportant, and the emission at high energies, made by the second order self-Compton emission, is never dominant. Therefore, even if a fraction of $\gamma$-rays get absorbed in $\gamma-\gamma$ collision producing pairs (likely with the X-ray emission from the disk corona), the spectrum is not strongly reprocessed, and the emission from the newly created pairs is not important enough to "fill the valley" between the two broad peaks of the blazar SED.

We do not know the relative fraction of time a jet spends producing blobs with large and small bulk Lorentz factors. Consider also that our current knowledge of blazars may be biased by the blazars observed (and detected) by EGRET. But, as mentioned in the introduction, EGRET detected only one fourth of the radio brightest blazars. This may suggest that a small value of the bulk Lorentz factor is the rule, not the exception, since in this regime the synchrotron emission is favoured with respect to the external Compton emission. The factor $\sim 20$ better sensitivity of GLAST with respect to EGRET will surely be crucial to solve this issue, and to shed light on these particular aspects of powerful relativistic jet.

Acknowledgements. This research has made use of the NASA/IPAC Extragalactic Database (NED), which is operated by the Jet Propulsion Laboratory, California Institute of Technology, under contract with the National Aeronautics and Space Administration. We acknowledge the EC funding under contract HPRCN-CT-2002-00321 (ENIGMA network).

\section{References}

Begelman, M. C., Rees, M. J., \& Sikora, M. 1994, ApJ, 429, 57 Fossati, G., Maraschi, L., Celotti, A., Comastri, A., \& Ghisellini, G. 1998, MNRAS, 299

Fuhrmann, L., Cucchiara, A., Marchili, N., et al. 2006, A\&A, 445, L1

Giommi, P., Blustin, A. J., Capalbi, M., et al. 2006 [arXiv:astro-ph/0606319]

Ghisellini, G., \& Madau, P. 1996, MNRAS, 280, 67

Ghisellini, G., Celotti, A., Fossati, G., Maraschi, L., \& Comastri, A. 1998, MNRAS, 301, 451

Ghisellini, G. 1999, Astron. Nachr., 320, 232

Guetta, D., Ghisellini, G., Lazzati, D., \& Celotti, A. 2004, A\&A, 421, 877

Hartman, R. C., Bertsch, D. L., Bloom, S. D., et al. 1999, ApJS, 123, 79

Krawczynski, H., Hughes, S., Horan, D., et al. 2004, ApJ, 601, 151

Pian, E., Foschini, L., Beckmann, V., et al. 2006, A\&A, 449, L21

Kardashev, N. S. 1962, Soviet Astron.-AJ, 6, 317

Lazzati, D., Ghisellini, G., \& Celotti, A. 1999, MNRAS, 309, L13

Meszaros, P. 2006, to appear in Reports on Progress in Physics [arXiv:astro-ph/0605208]

Rees, M. J. 1978, MNRAS, 184, 61

Sikora, M., Begelman, M. C., \& Rees, M. J. 1994, ApJ, 421, 153

Spada, M., Ghisellini, G., Lazzati, D., \& Celotti, A. 2001, MNRAS, 325, 1559

Tavecchio, F., Maraschi, L., Ghisellini, G., et al. 2002, ApJ, 575, 137

Villata, M., Raiteri, C. M., Balonek, T., J., et al. 2006, A\&A, 453, 817

Zhang, S., Collmar, W., \& Schönfelder, V. 2005, A\&A, 444, 767 